\title{
Fatores de risco biológicos e ambientais de crianças expostas ou não à poluição tabágica ambiental
}

Alice Stenzel de Pina Ferreira ${ }^{1}$, Fabiane Alves de Carvalho Ribeiro ${ }^{2}$, Cibelle Kayenne Martins Roberto Formiga ${ }^{3}$, Fabiana Pavan Viana ${ }^{4}$

${ }^{1}$ Enfermeira, Mestre em Atenção à Saúde. Goiânia, GO, Brasil. E-mail: stenzel.alice@gmail.com.

${ }^{2}$ Fisioterapeuta, Mestre em Ciências Ambientais e Saúde. Professor Assistente do Centro Universitário de Anápolis. Anápolis, GO, Brasil. E-mail: fabiane.carvalho@unievangelica.edu.br.

${ }^{3}$ Fisioterapeuta, Doutora em Ciências Médicas. Professora Adjunta da Universidade Estadual de Goiás. Goiânia, GO, Brasil. E-mail:

cibellekayenne@gmail.com.

${ }^{4}$ Fisioterapeuta, Doutora em Ciências Fisiológicas. Professora Adjunta da Pontifícia Universidade Católica de Goiás. Goiânia, GO, Brasil. E-mail: pavanviana@gmail.com.

Recebido: 08/06/2016. Aceito: 13/09/2017. Publicado: 31/12/2017.

Como citar esse artigo: Ferreira ASP, Ribeiro FAC, Formiga CKMR, Viana FP. Fatores de risco biológicos e ambientais de crianças expostas ou não à poluição tabágica ambiental. Rev. Eletr. Enf. [Internet]. 2017 [acesso em:

____19:a47. Disponível em: http://doi.org/10.5216/ree.v19.41695.

\section{RESUMO}

Objetivou-se investigar os fatores de riscos biológicos e ambientais de crianças expostas ou não à poluição tabágica ambiental (PTA). Estudo transversal, realizado com 670 crianças, de ambos os sexos, de oito a 12 anos em escolas de Anápolis (GO), com análise de questionário adaptado direcionado aos pais/responsáveis. Os pais do grupo de crianças não expostas à PTA (NEPTA) apresentaram maior escolaridade. O grupo de crianças expostas à PTA (EPTA) teve maior histórico de doença respiratória. O EPTA reside com um tabagista, comumente o pai, que fumam até 20 cigarros diários. O EPTA morava em residências com menos janelas, menor circulação de ar e maior registro de mofo. O EPTA apresenta mais doenças respiratórias e condições socioeconômicas desfavoráveis. Portanto, a exposição e o ambiente que vivem necessitam de maior cuidado. Profissionais de saúde e educadores devem promover a proteção, educação e estimular o abandono do tabagismo parental.

Descritores: Poluição por Fumaça de Tabaco; Desenvolvimento Infantil; Estudos Transversais.

\section{INTRODUÇÃO}

A poluição tabágica ambiental (PTA) pode predispor ou agravar o desenvolvimento de doenças tanto nos fumantes ativos, como nos expostos, pois contém três vezes mais nicotina, monóxido de carbono e até 50 vezes mais substâncias cancerígenas em relação à fumaça inalada pelo fumante ${ }^{(1)}$.

As crianças expostas à PTA apresentam alguns sintomas como: irritação ocular e nasal, cefaleia, dor de garganta e tosse. Contudo, a longo prazo podem apresentar: redução do crescimento e da função pulmonar, aumento da frequência de tosse, aumento da ocorrência de doenças respiratórias, agravamento da asma e 
distúrbios do sono ${ }^{(2)}$.

Deve-se levar em consideração que fatores, como a presença de mofo, poeira, pelos de animais e estiagem podem refletir na saúde dos envolvidos. Entretanto, não foram realizados estudos em âmbito nacional e internacional que documentam a relação existente entre os expostos ao tabaco e suas condições de moradia ${ }^{(3)}$.

Alguns aspectos podem estar relacionados à maior predisposição das crianças à PTA, como o baixo nível socioeconômico, a menor renda e escolaridade dos pais ${ }^{(4)}$, sendo assim, mais passíveis a exposição à PTA $^{(5)}$.

São escassos os trabalhos que investigam os efeitos da PTA em crianças de oito a 12 anos, assim como as possíveis associações do fumo passivo como a escolaridade dos pais, o histórico de sintomas e doenças respiratórias mais frequentes e as condições de moradia.

Considerando o tabagismo passivo como um problema de saúde pública devido as suas consequências, se faz necessário investigar os efeitos da fumaça do cigarro em crianças expostas à PTA e o objetivo deste estudo foi verificar os fatores de risco biológicos e ambientais de crianças expostas e não expostas à PTA.

\section{MÉTODOS}

Estudo transversal analítico realizado entre abril a junho de 2011 em crianças de oito a 12 anos (conforme Lei Federal ํㅗ 8.069/90, artigo 2 ㅇ que considera criança pessoa de até 12 anos incompletos) de escolas municipais de ensino fundamental da cidade de Anápolis, Goiás. Foram identificadas 11 escolas pela Secretaria Municipal de Educação, contudo oito instituições aceitaram participar da pesquisa.

Para definição dos participantes do estudo foram selecionados 670 crianças, voluntários, de ambos os sexos, compreendidos na faixa etária de oito a 12 anos incompletos e seus respectivos pais e/ou responsáveis legais, os quais participaram da pesquisa após a assinatura do Termo de Consentimento Livre e Esclarecido (TCLE) e que preencheram o questionário epidemiológico adaptado ${ }^{(6)}$ de forma completa. A escolha desta faixa etária foi justificada pelo fato de que crianças maiores de seis anos de idade, geralmente apresentam maior capacidade de compreensão necessária à realização da avaliação proposta, quando estimuladas e orientadas pelo examinador ${ }^{(7)}$.

As crianças que entregaram o questionário epidemiológico adaptado de forma incompleta, que faltaram aula no dia em foi explicado o estudo e distribuído o TCLE aos alunos, assim como aqueles que não compareceram no dia do exame físico foram excluídas da investigação. Foi considerado variável de exposição à PTA aquelas crianças que moravam com fumantes há pelo menos seis meses. E para caracterização do estudo foi considerado histórico de doenças respiratórias com relato de atendimento e diagnóstico médico para: asma, bronquite, pneumonia e rinite ${ }^{(6)}$.

Os voluntários foram classificados em dois grupos: escolares expostos à poluição tabágica ambiental (EPTA) e escolares não expostos à poluição tabágica ambiental (NEPTA).

O tamanho da amostra foi definido utilizando um nível de significância de 0,5\% e um poder de teste 
de $80 \%$, com margem de erro de 4,6\%, a estimativa mínima para o tamanho da amostra é de 454 participantes. Foram excluídos do estudo 215 (32\%) participantes devido ao preenchimento incompleto do questionário (Figura 1).

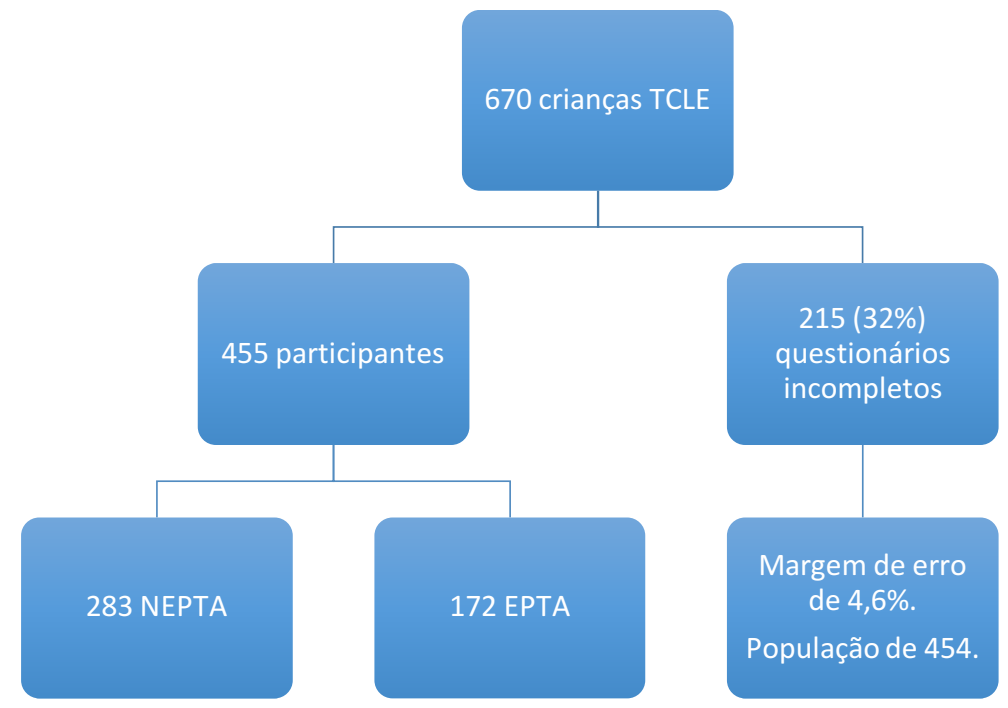

Figura 1: Fluxograma do número de participantes, crianças não expostas à poluição tabágica ambiental (NEPTA), crianças expostas à PTA (EPTA), indivíduos excluídos e margem de erro da população do estudo. Anápolis, GO, Brasil, 2011.

Para realização do estudo foi requerida a autorização à Secretaria Municipal de Educação de Anápolis, para o desenvolvimento da pesquisa nas escolas, bem como foi solicitada também a autorização das escolas. Nessas, foram contatados os responsáveis legais da instituição, assim como, os pais ou responsáveis, enviando-se um comunicado na agenda escolar do aluno, onde foi apresentado o Termo de Consentimento Livre e Esclarecido (TCLE) e o questionário epidemiológico adaptado ${ }^{(6)}$.

O questionário auto aplicado pelos pais/responsáveis continha os seguintes dados: informações de identificação; antecedentes familiares de doenças respiratórias; ocorrência de tabagismo materno durante a gestação; presença de mofo na residência, número de habitantes e número de cômodos em casa; escolaridade dos pais; presença de doença respiratória ou outros sintomas; presença de fumantes em casa; número de fumantes; tempo de convívio da criança com pessoas fumantes; período de duração do hábito tabágico; identificação das pessoas fumantes e tipo de fumo utilizado pelos tabagistas da casa ${ }^{(6)}$.

As crianças participantes foram avaliadas na escola, em local adequado pelos pesquisadores quanto ao peso com balança digital (marca: Geratherm), onde a criança deveria estar com roupas leves (bermuda e camiseta) e descalça.

Para a verificação da altura foi usado estadiômetro, fixado na parede, em local de piso regular. A criança estava descalça e com o mínimo de roupa possível para que se tornasse visível a posição de seu corpo. Ela foi posicionada em pé, de forma ereta, com os membros superiores pendentes ao longo do corpo, os calcanhares unidos e as pontas dos pés afastadas aproximadamente em $60^{\circ}$ entre si (o ajustamento dos pés depende dos joelhões estarem ou não em contato entre si), o peso corporal distribuído igualmente sobre ambos os pés e a cabeça orientada em paralelo com o solo. 
Posteriormente, foi calculado o índice de massa corporal (IMC) pela fórmula IMC = peso/altura ${ }^{2}$ e verificado o valor percentil pelo site: http://www.telessaudebrasil.org.br/apps/calculadoras/?page=7.

Ao final do estudo foi ministrado pelos pesquisadores palestras aos alunos e pais sobre tabagismo e seus malefícios.

Todo o conjunto de dados coletados nas avaliações foram organizados em uma planilha eletrônica do Excel ${ }^{\circ}$. Após a preparação de toda a planilha os dados foram transferidos para uma planilha do Statistical Package for the Social Sciences (SPSS) versão 15.0 e processadas as analises.

Inicialmente testou-se a normalidade das variáveis por meio do Teste Kolmogorov-Smirnov, para verificar a utilização de estatística paramétrica ou não paramétrica. Nas análises descritivas foram calculadas a média, mediana, desvio padrão (DP), valores mínimos e máximos para as variáveis contínuas e as frequências absoluta e relativa para as variáveis discretas.

Os dados foram avaliados pelo método de regressão logística, obtendo-se as razões de odds ratio (OR) e intervalos de confiança (IC) de todas as variáveis em todas as análises, utilizando-se um nível de significância de 0,05 .

Este trabalho foi aprovado pelo Comitê de Ética e Pesquisa (CEP) do Centro Universitário de Anápolis, Goiás, número interno do protocolo da CONEP/SISNEP CAAE: 0153/2009, Ofício de Aprovação 051/2010.

\section{RESULTADOS}

De acordo com os resultados obtidos não houve diferenças significativas entre o grupo NEPTA quando comparadas com o grupo EPTA em relação ao sexo, idade, peso, altura e IMC (Tabela 1).

Tabela 1: Características antropométricas das crianças não expostas à poluição tabágica ambiental (NEPTA=283) e expostos à poluição tabágica ambiental (EPTA=172) de escolas públicas de Anápolis, GO, Brasil, 2011.

\begin{tabular}{|c|c|c|c|c|c|c|c|c|}
\hline \multirow{2}{*}{ Escolares } & \multirow{2}{*}{$\mathbf{n}$} & \multirow{2}{*}{ M } & \multirow{2}{*}{ DP } & \multicolumn{2}{|c|}{ ICM 95\% } & \multirow{2}{*}{ p* } & \multirow{2}{*}{ OR (IC 95\%) } & \multirow{2}{*}{$\mathrm{p}^{* *}$} \\
\hline & & & & Min & Máx & & & \\
\hline \multicolumn{9}{|l|}{ Idade } \\
\hline NEFCA & 283 & 9,83 & 1,22 & 9,69 & 9,98 & & & \\
\hline EFCA & 172 & 9,95 & 1,31 & 9,76 & 10,15 & 0,323 & $1,08(0,93-1,26)$ & \\
\hline \multicolumn{9}{|l|}{ Peso } \\
\hline NEFCA & 283 & 37,48 & 10,33 & 36,28 & 38,69 & & & \\
\hline EFCA & 172 & 36,40 & 9,00 & 35,05 & 37,76 & 0,256 & $0,99(0,97-1,01)$ & 0,597 \\
\hline \multicolumn{9}{|l|}{ Altura } \\
\hline NEFCA & 283 & 141,76 & 9,16 & 140,69 & 142,84 & & & \\
\hline EFCA & 172 & 142,06 & 9,38 & 140,65 & 143,47 & 0,741 & $1,00(0,98-1,02)$ & 0,759 \\
\hline \multicolumn{9}{|l|}{ IMC } \\
\hline NEFCA & 283 & 58,71 & 30,80 & 55,11 & 62,32 & & & \\
\hline EFCA & 172 & 53,12 & 31,13 & 48,43 & 57,80 & 0,062 & $0,99(0,99-1,00)$ & 0,084 \\
\hline
\end{tabular}

Legenda: $\mathrm{n}$ : número total; M: média; DP: desvio padrão; ICM: intervalo de confiança da média; $\mathrm{p}^{*}$ : Teste Análise de Regressão Logística; OR: odds ratio; IC: intervalo de confiança; $\mathrm{p}^{* *}$ Kolmogorov-Smirnov.

Os pais do grupo NEPTA apresentaram maior grau de escolaridade $(p=0,005)$ que o EPTA. Já tabagismo materno durante a gestação $(p<0,001)$ foi maior no EPTA quando comparado com o NEPTA (Tabela 2 ). 
Tabela 2: Histórico familiar das crianças não expostas à poluição tabágica ambiental (NEPTA=283) e das expostas (EPTA=172) de escolas públicas de Anápolis, GO, Brasil, 2011.

\begin{tabular}{|c|c|c|c|c|}
\hline \multirow{2}{*}{ Variáveis } & NEPTA ( $\mathrm{N}=\mathbf{2 8 3})$ & EPTA (N=172) & \multirow{2}{*}{ OR (IC95\%) } & \multirow{2}{*}{$\mathbf{p}^{*}$} \\
\hline & n (\%) & $\mathrm{n}(\%)$ & & \\
\hline \multicolumn{5}{|l|}{ Escolaridade dos pais } \\
\hline Ensino Fundamental Incompleto & $77(27,2)$ & $70(40,7)$ & & \\
\hline Ensino Fundamental Completo & $152(53,7)$ & $78(45,3)$ & & \\
\hline Ensino Médio Incompleto & $54(19,1)$ & $24(14,0)$ & $0,67(0,50-0,89)$ & 0,005 \\
\hline \multicolumn{5}{|l|}{ Tabagismo materno durante a gestação } \\
\hline Sim & $13(4,6)$ & $36(20,9)$ & & \\
\hline Não & $270(95,4)$ & $136(79,1)$ & $5,50(2,82-10,71)$ & $<0,001$ \\
\hline \multicolumn{5}{|c|}{ Histórico de doença respiratória familiar } \\
\hline Sim & $84(29,7)$ & $72(41,9)$ & & \\
\hline Não & $199(70,3)$ & $100(58,1)$ & $1,71(1,15-2,53)$ & 0,008 \\
\hline \multicolumn{5}{|c|}{ Histórico de doença respiratória na criança } \\
\hline Sim & $56(19,8)$ & $106(61,6)$ & $6,51(4,26-9,95)$ & $<0,001$ \\
\hline Não & $227(80,2)$ & $66(38,4)$ & & \\
\hline \multicolumn{5}{|l|}{ Internação hospitalar } \\
\hline Sim & $5(1,8)$ & $12(7,0)$ & & \\
\hline Não & $278(98,2)$ & $160(93,0)$ & $4,17(1,44-12,05)$ & 0,008 \\
\hline \multirow{2}{*}{\multicolumn{5}{|c|}{$\begin{array}{l}\text { Histórico de doença respiratória na criança com necessidade } \\
\text { de atendimento médico }\end{array}$}} \\
\hline & & & & \\
\hline Não & $227(80,2)$ & $66(38,4)$ & & \\
\hline Rinite & $17(6,0)$ & $26(15,1)$ & $5,26(2,69-10,28)$ & $<0,001$ \\
\hline Asma & $1(0,4)$ & $14(8,1)$ & $48,15(6,22-372,99)$ & $<0,001$ \\
\hline Bronquite & $20(7,1)$ & $38(22,1)$ & $6,53(3,56-11,99)$ & $<0,001$ \\
\hline Pneumonia & $4(1,4)$ & $9(5,2)$ & $7,74(2,31-25,93)$ & 0,001 \\
\hline Outras & $5(1,8)$ & $3(1,7)$ & $2,06(0,48-8,86)$ & 0,330 \\
\hline Total & $56(19,8)$ & $106(61,6)$ & $6,51(4,26-9,95)$ & $<0,001$ \\
\hline
\end{tabular}

Legenda: p*: Teste de Regressão Binária Logística; OR: odds ratio; IC: intervalo de confiança.

O grupo EPTA teve maior histórico de doença respiratória familiar $(p=0,008)$ comparado ao NEPTA, assim como, as crianças do EPTA apresentaram maior histórico de doença respiratória $(p<0,001)$ em relação ao grupo NEPTA. Ademais foi encontrado que o grupo EPTA apresentou maior número de internações hospitalares $(p=0,008)$ quando comparado ao NEPTA (Tabela 2$)$.

Quanto aos hábitos tabágicos dos familiares do grupo EPTA, foi verificado que a maioria residia com apenas um tabagista $(77,3 \%)$, comumente o pai $(53,4 \%)$, que fumava até 20 cigarros convencionais por dia $(83,6 \%)$ e expunham as crianças por até seis horas diárias (50,6\%). Além disso, uma minoria fumava enquanto acompanhavam as crianças para a escola $(13,5 \%)$.

Em relação às condições de moradia, as crianças EPTA moravam em residências com menor número de janelas $(p=0,007)$ comparadas ao grupo NEPTA. Foi maior o registro da presença de mofo $(p=0,002)$ nas casas do grupo EPTA que no grupo NEPTA. E o grupo EPTA apresentou menor relato de boa circulação de ar $(p=0,042)$ comparado ao grupo NEPTA (Tabela 3$)$. 
Tabela 3: Distribuição das condições de moradia das crianças não expostas à poluição tabágica ambiental (NEPTA=283) e das expostas (EPTA=172) de escolas públicas de Anápolis, GO, Brasil, 2011.

\begin{tabular}{|c|c|c|c|c|}
\hline \multirow{2}{*}{ Variáveis } & NEPTA ( $\mathrm{N}=283$ ) & EPTA (N=172) & \multirow{2}{*}{ OR (IC95\%) } & \multirow{2}{*}{$\mathrm{p}^{*}$} \\
\hline & $\mathrm{n}(\%)$ & n (\%) & & \\
\hline \multicolumn{5}{|l|}{ Cômodos em casa } \\
\hline Até 3 & $10(3,5)$ & $13(7,6)$ & & \\
\hline$>3$ & $273(96,5)$ & $159(92,4)$ & $0,44(0,19-1,04)$ & 0,063 \\
\hline \multicolumn{5}{|l|}{ Janelas em casa } \\
\hline Até 3 & $30(10,6)$ & $34(19,8)$ & & \\
\hline$>3$ & $253(89,4)$ & $138(80,2)$ & $0,48(0,28-0,82)$ & 0,007 \\
\hline \multicolumn{5}{|l|}{ Boa circulação de ar } \\
\hline Sim & $250(88,3)$ & $140(81,4)$ & & \\
\hline Não & $33(11,7)$ & $32(18,6)$ & $1,73(1,02-2,93)$ & 0,042 \\
\hline \multicolumn{5}{|l|}{ Casa possui mofo } \\
\hline Sim & $72(25,4)$ & $68(39,5)$ & & \\
\hline Não & $211(74,6)$ & $104(60,5)$ & $0,52(0,34-0,78)$ & 0,002 \\
\hline \multicolumn{5}{|l|}{ Pessoas em casa } \\
\hline Até 2 & $176(62,2)$ & $95(55,2)$ & & \\
\hline$>2$ & $107(37,8)$ & $77(44,8)$ & $1,33(0,90-1,95)$ & 0,143 \\
\hline \multicolumn{5}{|l|}{ Possui animal } \\
\hline Sim & $114(40,3)$ & $62(36,0)$ & $0,83(0,56-1,23)$ & 0,369 \\
\hline Não & $169(59,7)$ & $110(4,0)$ & & \\
\hline
\end{tabular}

\section{DISCUSSÃO}

A exposição a poluição tabágica ambiental é um problema de saúde pública e tem sido associado a fatores culturais e socioeconômicos, assim como a comportamentos de risco à saúde ${ }^{(8)}$. Na presente pesquisa que os pais do grupo NEPTA apresentaram maior grau de escolaridade que o EPTA $(O R=0,67 ;$ IC95\%=0,50$0,89)$, dados semelhantes aos dos resultados encontrados por outro estudo ${ }^{(9)}$, foi visto que existe maior tendência para o hábito tabágico nos familiares de crianças com menor escolaridade quando comparado com àqueles que não fumam.

Por outro lado, existe uma grande variação socioeconômica entre os tabagistas no mundo ${ }^{(4)}$. A maior prevalência do tabagismo em indivíduos com menor escolaridade se deve possivelmente ao menor acesso a informação sobre os malefícios do uso do tabaco. Durante décadas no Brasil, as propagandas publicitárias estimularam o consumo do tabaco, impondo uma imagem de que aqueles que fumavam tinham maior virilidade, força, poder, independência, liberdade e autoafirmação ${ }^{(10)}$.

Ademais, verificou-se que a maioria das mães que fumaram na gestação tem cinco vezes mais chance de manterem o hábito tabágico $\left(\mathrm{OR}=5,50\right.$; IC95\%=2,82-10,71), como já é conhecido na literatura ${ }^{(11)}$. O tabagismo é um dos hábitos mais difíceis de abdicar, pois quanto maior o grau de dependência à nicotina, maior é a dificuldade enfrentada para o abandono do cigarro.

Por outro lado, alguns investigadores atribuem à continuidade do hábito tabágico gestacional devido ao estímulo do companheiro na permanência deste hábito ${ }^{(11)}$. 0 hábito tabágico gestacional está diretamente relacionado ao menor peso dos recém-nascidos e sobrepeso/obesidade na infância ${ }^{(12)}$. A continuidade deste hábito durante a amamentação e nos primeiros anos de vida pode acarretar o desenvolvimento de diversas doenças, como asma, bronquite, pneumonia, rinite, distúrbios do sono e 
problemas comportamentais ${ }^{(2)}$.

Já é comprovado que o hábito tabágico durante a gestação é prejudicial para a saúde dos neonatos, sendo assim, se faz necessário ações mais concretas durante o pré-natal voltadas para o combate do tabagismo materno ${ }^{(13)}$.

Na presente pesquisa, os familiares dos tabagistas apresentaram quase duas vezes mais chance de ter histórico de doenças respiratórias $(O R=1,71 ; \mathrm{IC} 95 \%=1,15-2,53)$ quando comparados aos não expostos à PTA.

É incontestável que a exposição à fumaça do cigarro cause doenças, porém o seu mecanismo ainda é desconhecido ${ }^{(2)}$. Assim como o tabagista, o fumante passivo, também está exposto à várias doenças tabacorelacionadas, tendo como principais as doenças crônicas não transmissíveis (DCNT), como os cânceres, as doenças cardiovasculares, o diabetes, as doenças respiratórias obstrutivas crônicas (DPOC) e entre outras ${ }^{(14)}$.

Pesquisa realizada na Espanha ${ }^{(15)}$ também observou que crianças expostas à PTA apresentam seis vezes mais chance de ter histórico de doenças respiratórias quando comparada às não expostas $(O R=6,51$; IC95\%=4,26-9,95).

As crianças são mais vulneráveis a exposição à PTA, pois são sujeitas com maior frequência no ambiente doméstico, nas escolas, locais públicos e em veículos de transporte ${ }^{(5)}$. É evidente que as crianças expostas apresentam o sistema imunológico deprimido, tornando-as propensas a desenvolver uma série de doenças como resfriados, infecções do ouvido médio, doenças respiratórias bem como pneumonia, bronquite e agravamento da asma ${ }^{(16)}$. Pesquisa recente ${ }^{(17)}$ verificou que crianças com histórico de doenças respiratórias constituem um grupo de alto risco para desenvolver doenças respiratórias agudas baixas e esse risco tende a aumentar com a exposição contínua da PTA.

No presente estudo verificou-se que as crianças expostas à PTA tiveram maior predisposição para desenvolver doenças respiratórias, tendo cinco vezes mais chance de ter rinite $(O R=5,26 ; \operatorname{IC} 95 \%=2,69-10,28)$, 48 vezes mais chance de ter asma $(\mathrm{OR}=48,15 ; \mathrm{IC} 95 \%=6,22-372,99)$, seis vezes mais chance de ter bronquite $(O R=6,53$; IC95\%=3,56-11,99) e sete vezes mais chance de ter pneumonia $(O R=7,74 ;$ IC95\%=2,31-25,93).

Fumantes ativos e passivos têm risco de 20 a 30 vezes de adquirir as doenças acima $\operatorname{citadas}^{(9)}$. 0 fumante ativo consome a menor parte da fumaça do tabaco, enquanto o tabagista passivo consome cerca de $85 \%$ da fumaça dispersada pelo cigarro e ainda a que sai da boca do fumante ${ }^{(18)}$.

O Instituto Nacional de Câncer (INCA) refere que a poluição tabágica ambiental contém cerca de 4.700 substâncias tóxicas ao organismo, como o monóxido de carbono, amônia, cetonas, formaldeído, além de outras 43 cancerígenas, tais como: arsênio, níquel, benzopireno, cádmio, chumbo, resíduos de agrotóxicos e substâncias radioativas ${ }^{(1)}$.

Em conformidade com os resultados encontrados por outros autores da literatura ${ }^{(2,15-16)}$ os tabagistas passivos têm quatro vezes mais chance de sofrer internações hospitalares por doenças respiratórias quando comparado com os não tabagistas passivos (OR=4,1; IC95\%=1,44-12,05). Os custos de internações por doenças tabaco-relacionadas chegam a 1,5 bilhão de reais, sendo $11 \%$ dos registros documentados ${ }^{(19)}$. Deste modo torna-se imprescindível que as campanhas antitabagismo promovidas pelo Ministério da Saúde por 
meio do INCA nos estados, cujas ações articuladas compõem o Programa Nacional de Controle do Tabagismo (PNCT) executem atos efetivos e concretos de controle do tabagismo ativo e proteção aos expostos principalmente em suas residências.

As leis antitabagismo em vigor no Brasil tem alcançado sucesso na mudança de hábitos sociais, na redução do lucro da indústria do tabaco e redução no consumo de cigarros, contudo ainda não protege de forma efetiva os expostos a fumaça do cigarro ambiental.

No presente estudo foi encontrado resultado semelhante ao da literatura ${ }^{(20-21)}$ em que a criança exposta à PTA convive com um tabagista, comumente o pai, que consome até 20 cigarros convencionais por dia $^{(10-15)}$. Isso pode ser explicado em parte, segundo a Organização Mundial de Saúde (OMS), pela maior prevalência do hábito tabágico por homens ${ }^{(1)}$.

Pesquisa realizada em Portugal ${ }^{(21)}$ também relatou elevado número de cigarros fumados por dia. Apesar do alto número de cigarros consumidos, esse percentual vem decaindo ${ }^{(10,15)}$. Comparado a outros países. Essa redução pode ser explicada devido as campanhas antitabagismo, as leis que proíbem o fumo em ambientes fechados como o Decreto lei no 5.658 de 02 de janeiro de 2008 que promulga a ConvençãoQuadro sobre o Controle do Tabaco, a diminuição da propaganda e fiscalização efetiva ${ }^{(9)}$.

Quanto ao período de exposição à PTA, esse foi elevado, cerca de seis horas diárias. Dado semelhante foi encontrado em outro estudo português ${ }^{(20)}$, demonstrando, assim, que o tabagismo passivo é muito comum em nossa sociedade, consequentemente um problema de saúde pública devido as consequências da exposição à PTA.

Quanto às condições de moradia, os achados do presente estudo se assemelham a de outra pesquisa ${ }^{(7)}$, que verificou que crianças não expostas à PTA têm mais chances de viver em casas com mais janelas $(O R=0,48 ; I C 95 \%=0,28-0,82)$, consequentemente, menor chance das casas apresentarem mofo $(O R=0,52$; IC95\%=0,34-0,78). Ao contrário, as crianças expostas à PTA tinham quase duas vezes mais chances de viver em casas com menor circulação de ar (OR=1,73; IC95\%=1,02-2,94).

São escassos os estudos que investigam as condições de moradia de crianças expostas à PTA, contudo, um estudo australiano ${ }^{(22)}$ identificou que não é apenas a PTA o principal causador de doenças respiratórias. Outros fatores ambientais foram identificados em um estudo brasileiro ${ }^{(3)}$ como a presença de poeira, pelos de animais domésticos, ácaros, mofo aparente nas paredes, estiagem, fumaça da queima da cana de açúcar e poluentes ambientais, uma vez que alteram de forma negativa a qualidade do ar e favorecem o desenvolvimento de doenças respiratórias. Possivelmente este fato está relacionado com a baixa renda familiar, na qual dificulta a construção de casas arejadas, que por sua vez, impede a circulação de ar e aumenta a PTA.

Os profissionais da área da saúde devem receber treinamento específico para saber lidar com o atendimento deste público, no intuito de estimular a cessação do tabagismo materno durante a gestação e dos demais integrantes da família ${ }^{(2)}$, visto que o hábito de um pode influenciar os outros. Uma possível estratégia é a realização de campanhas e acompanhamento individualizado dos envolvidos ${ }^{(23)}$, pela equipe 
da Estratégia Saúde na Família (ESF). A ESF, dentro da Atenção Primária à Saúde (APS) é a porta de entrada para movimentos e ações individuais e coletivos que englobam a promoção à saúde até a reabilitação.

Dentro desse contexto o Programa Nacional de Controle do Tabagismo (PNCT) oferece apoio e tratamento para a cessação do tabagismo com ações educativas e acompanhamento individual e coletivo nos Núcleos de Apoio Saúde da Família (NASF), contudo a realidade é complexa, exigindo assim que as equipes atuem de forma empática com a população tabagista visando à adesão ao tratamento e orientações oferecidas.

\section{CONCLUSÃO}

Existem vários estudos sobre os efeitos da PTA em crianças com doenças do trato respiratório, mas são poucos os estudos voltados para as condições de moradia de crianças expostas à PTA. No presente estudo as mesmas apresentaram mais doenças respiratórias crônicas, o pai foi o principal tabagista a expor a criança à PTA e viviam em ambientes com pouca circulação de ar.

Portanto, o tabagismo passivo é um problema de saúde pública com particular impacto na saúde das crianças. Não apenas a exposição deve ser levada em consideração, mas o ambiente em que vivem as crianças necessita de maior atenção e cuidado por parte dos pais/responsáveis. Os profissionais de saúde, juntamente com os educadores devem proporcionar mais informações aos pais sobre os malefícios da exposição à PTA pelas crianças, instigar a adesão de programas de abandono do tabagismo parental e promover a promoção e proteção à saúde de todos os envolvidos.

Os resultados deste estudo contribuíram para compreender melhor o contexto em que vivem as crianças expostas à PTA e as doenças associadas, mas encontrou limitações condicionadas ao questionário que não avaliou a renda familiar e os dados fornecidos pelos pais/responsáveis sujeitos a viés de memória e problemas com a interpretação.

\section{Financiamento}

Essa pesquisa foi financiada pela Fundação de Amparo a Pesquisa do Estado de Goiás (FAPEG), processo número: 200910267000516.

\section{REFERÊNCIAS}

1. Instituto Nacional de Câncer José Alencar Gomes da Silva. Tabagismo [Internet]. Rio de Janeiro: INCA/Ministério de Saúde; c1996-2017 [acesso em: 31 dez. 2017]. Disponível em:

http://www2.inca.gov.br/wps/wcm/connect/acoes_programas/site/home/nobrasil/programa-nacional-controletabagismo/tabagismo.

2. Yang S, Decker A, Kramer MS. Exposure to parental smoking and child growth and development: a cohort study. BMC Pediatr [Internet]. 2013 [acesso em: 31 dez. 2017];13(1):104. Disponível em: https://doi.org/10.1186/1471-243113-104. 
3. Ribeiro FAC, Moraes MKR, Caixeta JCM, Silva JN, Lima AS, Parreira SLS, et al. Percepção dos pais a respeito do tabagismo passivo na saúde de seus filhos: um estudo etnográfico. Rev Paul Pediatr [Internet]. 2015 [acesso em: 31 dez. 2017];33(4):394-9. Disponível em: http://doi.org/10.1016/j.rpped.2015.02.003.

4. Douglas L, Szatkowski L. Socioeconomic variations in access to smoking cessation interventions in UK primary care: insights using the Mosaic classification in a large dataset of primary care records. BMC Public Health [Internet]. 2013 [acesso em: 31 dez. 2017];13(1):546. Disponível em: http://doi.org/10.1186/1471-2458-13-546.

5. Quiroz-Arcentales L, Hernández-Flórez LJ, Agudelo Calderón CA, Medina K, Robledo-Martínez R, Osorio-García SD. Enfermedad y síntomas respiratorios en niños de cinco municipios carboníferos del Cesar, Colombia. Rev Salud Pública [Internet]. 2013 [acesso em: 31 dez. 2017];15(1):66-79. Disponível em:

https://www.scielosp.org/article/rsap/2013.v15n1/66-79/es/?abstract_lang=en.

6. Carvalho LMT, Pereira EDB. Morbidade respiratória em crianças fumantes passivas. J Pneumol [Internet]. 2002 [acesso em: 31 dez. 2017];28(1):8-14. Disponível em: http://doi.org/10.1590/S0102-35862002000100004.

7. Rodrigues JC, Cardieri JMA, Bussamra MHCF, Nakaie CMA, Almeida MB, Silva Filho LVF, et al. Provas de função pulmonar em crianças e adolescentes. J Pneumol [Internet]. 2002 [acesso em: 31 dez. 2017];28(Supl 3):S207-21. Disponível em: http://www.luzimarteixeira.com.br/wp-content/uploads/2010/07/provas-de-funcao-pulmonar-emcriancas-e-adolescentes.pdf.

8. Sun L-Y, Cheong H-K, Lee E-W, Kang K-J, Park J-H. Affecting Factors of Secondhand Smoke Exposure in Korea: Focused on Different Exposure Locations. J Korean Med Sci [Internet]. 2016 [acesso em: 31 dez. 2017];31(9):1362. Disponível em: http://doi.org/10.3346/jkms.2016.31.9.1362.

9. Malta DC, Oliveira TP, Vieira ML, Almeida L, Szwarcwald CL. Uso e exposição à fumaça do tabaco no Brasil: resultados da Pesquisa Nacional de Saúde 2013. Epidemiol e Serviços Saúde [Internet]. 2015 [acesso em: 31 dez. 2017];24(2):239-48. Disponível em: http://doi.org/10.5123/S1679-49742015000200006.

10. Malta DC, Oliveira TP, Luz M, Stopa SR, Silva Junior JB, Reis AAC. Smoking trend indicators in Brazilian capitals, 2006-2013. Cien Saude Colet [Internet]. 2015 [acesso em: 31 dez. 2017];20(3):631-40. Disponível em:

http://doi.org/10.1590/1413-81232015203.15232014.

11. Poletta FA, López-Camelo JS, Gili JA, Montalvo G, Castilla EE. Consumo y exposición al humo de tabaco en mujeres embarazadas de Ecuador. Rev Panam Salud Pública [Internet]. 2010 [acesso em: 31 dez. 2017];27(1):56-65. Disponível em: https://www.scielosp.org/article/rpsp/2010.v27n1/56-65/es/?abstract_lang=en.

12. Muraro AP, Gonçalves-Silva RMV, Ferreira MG, Silva GA, Sichieri R. Effect of the exposure to maternal smoking during pregnancy and childhood on the body mass index until adolescence. Rev Saude Publica [Internet]. 2015 [acesso em: 31 dez. 2017];49:41. Disponível em:: http://doi.org/10.1590/S0034-8910.2015049005423.

13. Griffis H, Matone M, Kellom K, Concors E, Quarshie W, French B, et al. Home visiting and perinatal smoking: a mixed-methods exploration of cessation and harm reduction strategies. BMC Public Health [Internet]. 2016 [acesso em: 31 dez. 2017];16:764. Disponível em: http://doi.org/10.1186/s12889-016-3464-4.

14. Pinto MT, Pichon-Riviere A, Bardach A. Estimativa da carga do tabagismo no Brasil: mortalidade, morbidade e custos. Cad Saude Publica [Internet]. 2015 [acesso em: 31 dez. 2017];31(6):1283-97. Disponível em:

http://doi.org/10.1590/0102-311X00192013.

15. Gonzalez-Barcala FJ, Pertega S, Sampedro M, Lastres JS, Gonzalez MA, Bamonde L, et al. Impact of parental smoking on childhood asthma. J Pediatr (Rio J) [Internet]. 2013 [acesso em: 31 dez. 2017];89(3):294-9. Disponível em: http://doi.org/10.1016/j.jped.2012.11.001.

16. Coelho SA, Rocha SA, Jong LC. Consequências do tabagismo passivo em crianças. Ciência, Cuidado e Saúde [Internet]. 2012 [acesso em: 31 dez. 2017];11(2):294-301. Disponível em:

http://doi.org/10.4025/cienccuidsaude.v11i2.10281.

17. Sigaud $\mathrm{CH}$, Castanheira $\mathrm{AB}$, Costa $\mathrm{P}$. Association between secondhand smoking in the home and respiratory morbidity in preschool children. Rev Esc Enferm USP [Internet]. 2016 [acesso em: 31 dez. 2017];50(4):562-8. Disponível em: http://doi.org/10.1590/S0080-623420160000500004.

18. Ministério da Saúde, Secretaria de Atenção à Saúde, Departamento de Atenção Básica. Manual de Telessaúde para a Atenção Básica / Atenção Primária à Saúde: protocolo de solicitação de teleconsultorias. Brasília: Ministério da Saúde, 2013 [acesso em: 31 dez. 2017]. Disponível em:

http://bvsms.saude.gov.br/bvs/publicacoes/manual telessaude_atencao basica.pdf.

19. Sousa-Carmo SVT, Vilar MCH, Moraes MA. Gastos hospitalares de doenças tabaco-relacionadas no Sistema Único de Saúde - SUS: um estudo comparativo entre o Brasil, Região Sudeste e estado de São Paulo no ano de 2013. BEPA,

Rev. Eletr. Enf. [Internet]. 2017 [acesso em:_____];19:a47. Disponível em: http://doi.org/10.5216/ree.v19.41695. 
Bol. epidemiol. paul. (Online) [Internet]. 2015 [acesso em: 31 dez. 2017];12(140):3-14. Disponível em:

http://www.saude.sp.gov.br/resources/ccd/homepage/bepa/edicao-2015/edicao_140_-_agosto_2.pdf.

20. Pereira AM, Morais-Almeida M, Sousa AS, Jacinto T, Azevedo LF, Cordeiro CR, et al. Prevalência da exposição ao fumo ambiental do tabaco em casa e do tabagismo na população Portuguesa - o estudo INAsma. Rev Port Pneumol [Internet]. 2013 [acesso em: 31 dez. 2017];19(3):114-24. Disponível em::

https://doi.org/10.1016/j.rppneu.2013.01.002.

21. Precioso J, Samorinha C, Araújo C, Macedo M, Antunes H. Exposición de ninhos al humo ambiental del tabaco (FAT): um estúdio efectuado con alunos de 4ำ curso de escolaridade de Braga - Portugal. Prev Tab [Internet]. 2011 [acesso em: 31 dez. 2017];13(3):112-6. Disponível em:

https://repositorium.sdum.uminho.pt/bitstream/1822/15722/1/Exposicion\%20de\%20ninos\%20al\%20humo\%20ambie ntal\%20del\%20tabaco\%20-\%20FAT.pdf.

22. Simpson JL, Guest M, Boggess MM, Gibson PG. Occupational exposures, smoking and airway inflammation in refractory asthma. BMC Pulm Med [Internet]. 2014 [acesso em: 31 dez. 2017];14:207. Disponível em:

http://doi.org/10.1186/1471-2466-14-207.

23. Bartsch A-L, Härter M, Niedrich J, Brütt AL, Buchholz A. A Systematic Literature Review of Self-Reported Smoking Cessation Counseling by Primary Care Physicians. PLoS One [Internet]. 2016 [acesso em: $31 \mathrm{dez}$.

2017];11(12):e0168482. Disponível em: https://doi.org/10.1371/journal.pone.0168482. 